des ersten Buches die Christenverfolgungen abgeschlossen waren (s. Bd. I S. 19, $3 \dot{\varepsilon} \mu \varepsilon \lambda \dot{\varepsilon} \tau \eta \sigma \alpha v$ ) und Eusebius dasselbe noch im zwölften Buch (Kap. 10,7) konstatiert, dagegen im 13. (Kap. 6, 12) einen Wiederausbruch derselben für möglich hält (was auf die Verschlechterung der Beziehungen des Licinius zu Konstantin und das immer christenfeindlichere Verhalten jenes Kaisers zu Beginn der zwanziger Jahre des vierten Jahrhunderts hinweist), hat Eusebius mit der Abfassung der P. E. offenbar 312 begonnen und mindestens 10 Jahre daran gearbeitet.

\title{
Eusebius und die von ihm zitierten Autoren
}

Aus seinem Verhalten gegen die noch vorhandenen Werke können wir einen Schluß auf sein Vorgehen gegenüber verlorenen Schriften ziehen. Bei der Unmasse der Zitate in der P. E. sind ihm nur folgende Verstöße unterlaufen: Clem. Al. prot. $\mu \varepsilon \tau^{\prime} \dot{\lambda} \lambda i^{\prime} \alpha^{1}$ ) (in Wahrheit früher): Bd.I S. 92,11; strom. 6. Buch (richtig 5.): Bd. II S. 56, I; Plat. resp. 3. B. (statt 4. B.): Bd. II S. 129, 3;

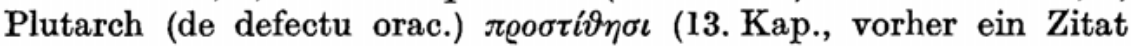
aus dem 17. Kap.) Bd. I S. 227, 19; Philo Iud. ėлı $\varepsilon^{\prime} \gamma \varepsilon \iota$ (steht in Wahr-

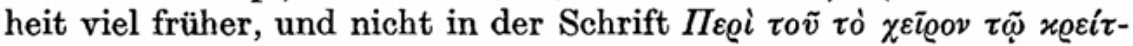

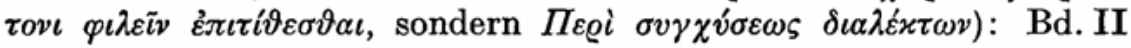
S. 36,14 und $24^{2}$ ). Das ist alles.

Die Zitate selber sind genau und wörtlich ${ }^{3}$ ); entsprechend seinen

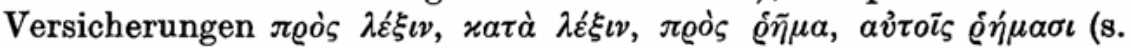
das Wortregister $)^{4}$ ). Nur gelegentlich finden sich geringe stilistische Änderungen am Anfang der Zitate, wie sie eben der Zusammen-

1) Zur Bezeichnung späterer Stellen bereits zitierter Werke gebraucht Eus. stereotype, aber mannigfach variierte Wendungen, die Dindorf I S. XIX

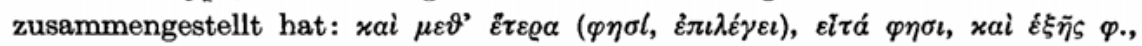

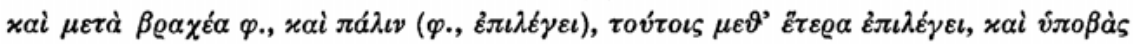

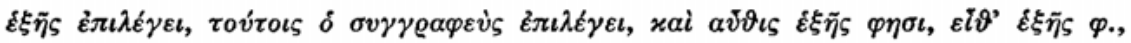

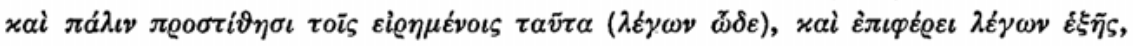

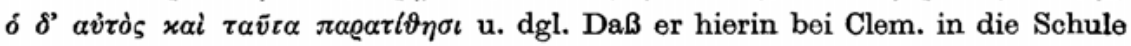
gegangen ist, lehrt z. B. Bd. II S. 203, 1.

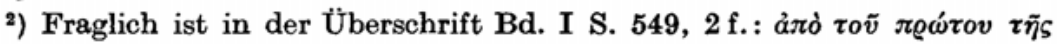

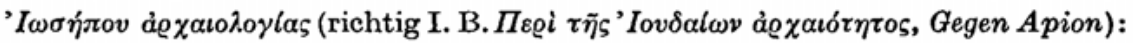
die ganze Überschrift fehlt ja in der Hs. I. Dieselbe falsche Angabe steht (aber nur in I) auch Bd. I S. 483, $17 \mathrm{f}$.

3) Nur beiDiodor sah er sich zu einigenÄnderungen genötigt, s. weiter unten.

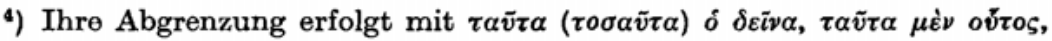
$\tau \alpha \tilde{v} \tau \alpha \mu \dot{\varepsilon} \nu$ ov̀v $\tilde{\omega} \delta \varepsilon \varepsilon \dot{\varepsilon} \chi \dot{\varepsilon} \tau \omega$ u. dgl. 
hang mit dem Ganzen, dem sie eingefügt sind, erfordert. Um so unbegreiflicher sind daher die Verunglimpfungen, die Eusebius von Gelehrten früherer Zeit erfahren hat (man mag sie bei J. Freudenthal, Hellenistische Studien ${ }^{1}$ ), Heft 1, S. 3f. nachlesen, darunter das ebenso kecke wie unbegründete Urteil Niebuhrs). Von philologischer Seite war Valckenaer der erste, der Eusebius Recht zuteil werden ließ ${ }^{2}$ ). Ihm stimmte Dindorf bei (s. seine Ausgabe I S. XVIIIf.), ebenso Freudenthal a.a. O. S. 4-14 und S.,184, und kein Unbefangener kann anders urteilen. Eusebius vermeidet es, die zitierten Texte seinem eigenen Stil anzugleichen. Wie gewissenhaft er vorgeht, lehrt der Vergleich mit Clemens. Zwei Stellen sind besonders bezeichnend: Bd. I S. 493,11-18 zitiert er eine Stelle des Aristobul aus Clem., Bd. II S. 190, 18-191, 4f. dieselbe Stelle

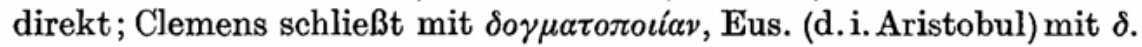

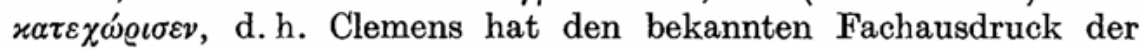
hellenistischen Zeit $\chi \alpha \tau \alpha \chi \omega \varrho i \zeta \varepsilon v v$, weil er seinen attizistischen Tendenzen zuwider war, weggelassen, Eus. bewahrt (s. Mras, Rheinisches Museum N. F. $\left.92[1944]^{221}\right)^{3}$ ). Auf eine andere Stelle hat Freudenthal a. a. O. S. 13 hingewiesen, Bd. I S. 522, 17-25 (Artapanus) im Vergleich mit Clem. Al. strom. I 154, 2f.: Clem. kürzt stark und gibt nur den ungefähren Sinn, und - was besonders bezeichnend

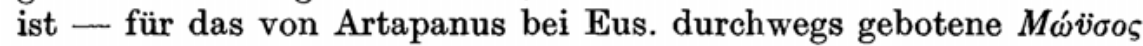

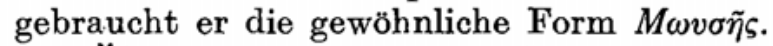

Über die Zitate aus Diodor, Dionys von Halikarnaß, Plato, Porphyrius, Xenophon und aus der Bibel hat Heikel einiges gesagt (S. 56-69: Quae ratio inter codices Eusebii et codices eorum scriptorum, e quibus nonnulla excerpsit Eusebius, intersit). Darüber ließe sich und müßte man viel mehr sagen, als Heikel gesagt hat, auch müßten alle von Eus. zitierten Autoren in die Untersuchung einbezogen werden, wozu hier natürlich nicht der Platz ist. Nur zu den Exzerpten aus Diodor will ich einiges bemerken. Im ersten, zweiten und dritten Buch stand Eus. vor der Aufgabe, aus Diodors umfangreichem Schrifttum die passende Auswahl zu treffen. Er hat diese Aufgabe mit Geschick gelöst, wobei es freilich nicht ohne zum Teil starke Kürzung und Veränderung

1) Alexander Polyhistor und die von ihm erhaltenen Reste jüdischer und samaritanischer Geschichtswerke (Jahresber. des jüd.-theol. Seminars, Breslau 1874 und 1875).

$\left.{ }^{2}\right)$ Diatribe de Aristobulo (wieder abgedruckt in Gaisfords Ausgabe) 75 (IV 408 Gaisf.) und 82 (IV $413 \mathrm{f}$. Gaisf.).

a) Daraus folgt, daß der echte Wortlaut des Aristobul hier bei Eus. vorliegt. 
abgehen konnte, gelegentlich auch nicht ohne kleine Ungenauigkeiten, so Bd. I S. 69, 9f. (s. meine Anmerkung zu dieser Stelle), S. 71, 12f. (bei Diodor IV 31, 4 heißt es: weil Herakles den Sohn des Eurytos tückisch von einem Turm hinabgestoßen hatte, grämte er sich hinterher) und S. 75, 7 (s. meine Anmerkung). Hervorgehoben zu werden verdient: Diodor stimmt öfter mit Eus. Hs. A als mit BONV überein; Eus. bietet S. 27, 1 mit Diodors Hss. CFGLM die erweiterte Fassung (gegen Diodors Hss. ABDEN); S. 34, 25 Eus. $\pi \alpha ́ v \tau \eta$ mit Diodors Hss. CF (die anderen Hss. $\pi \alpha v \tau \alpha \chi \tilde{\eta}$ ); S. 68, 1 Eus. $\zeta \tilde{v} \vartheta o v$ mit denselben Hss. Diodors (die anderen $\zeta \tilde{v} \vartheta \circ \varsigma$ ); S. 72, 5 Eus. $\pi \rho \tilde{\omega} \tau o v$

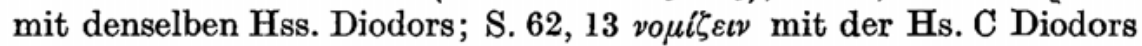
(s. zur Stelle); S. 112, 23 = Diod. Hss. CD; zwei andere Namen als bei Diodor S. 72, 15 .

Zwei Stellen aus Plato habe ich im Rheinischen Museum N. F. 92 (1944) 218f. behandelt. Die Tatsache, daß Eusebius manche Stelle bei Plato (bes. in den Gesetzen mit ihrer vertrackten Sprache) andersals wir verstanden (womit übrigens nicht gesagt sein soll, da $\beta$ wir diese alle richtig verstehen) oder auch mißverstanden hat, darf nicht zur Änderung solcher Stellen verführen. Auf zwei bemerkenswerte Abweichungen von unserem platonischen Text weise ich hier hin:

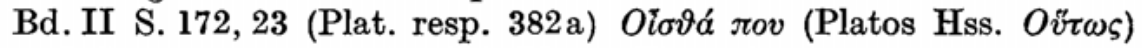

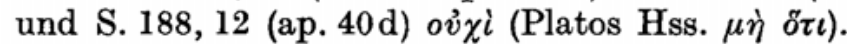

Merkwürdig ist, daß Eus. trotz der guten Disposition der P. E. sowie seiner zahlreichen Vor- und Rückverweisungen (s. das Namenund Sachregister unter Eusebius) bei mehrfach wiederholten Zitaten Hinweise auf Stellen, an denen sie bereits vorgekommen sind, unterläßt; so wird z. B. Plat. Tim. 41 ab Bd. II S. 69, 9ff. und S. 243, $1 \mathrm{ff}$. von Eus. direkt aus Plato und S. 207, 16 indirekt (aus Clem.) angeführt, nirgends aber vermerkt, daß das Zitat bereits vorgekommen ist.

Woher hat sich Eusebius seine Zitate verschafft? Die Antwort lautet: aus der reichen bischöflichen, von Pamphilos gegründeten Bibliothek von Caesarea in Palästina ${ }^{1}$ ), deren Grundstock die von Origenes bei seiner Flucht nach Caesarea dorthin gebrachten Bücher waren. In dieser fehlten zwar Tragiker, Komiker ${ }^{2}$ ) und Lyriker, desgleichen wohl auch die Originalschriften der Stoiker und Epikureer, dafür aber war sie sehr reich an Werken der Historiker und

1) S. Eus. hist. eccl. VI 32, 3. Diese Bibliothek würde eine eigene Untersuchung verdienen.

$\left.{ }^{2}\right)$ Keinen einzigen Tragiker- oder Komikervers hat Eus. in der P.E. direkt zitiert. Wie viele Belege für seine Ausführungen hätte er bei Euripides oder Menander finden können! 
späterer Platoniker ${ }^{1}$ ). Überhaupt hat Freudenthal mit seiner Behauptung (a.a.O. S. 7) recht: ,Eusebios ist nämlich ebenso belesen in der neueren, wie wenig bewandert in der älteren Litteratur der Griechen " (außer Plato und Xenophon). Dieses große Exzerptenwerk hätte ohne Mithilfe von amanuenses kaum zustande kommen können. Man tut m. E. am besten, sich folgende Vorstellung davon zu machen: Eus. sitzt auf seiner $x a \vartheta \varepsilon \delta \varrho \alpha$, umgeben von seinen $\delta \iota \alpha ́ x o v o \iota$, die zugleich

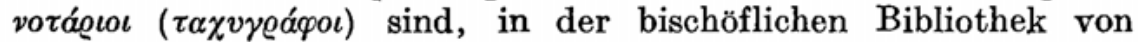
Caesarea. Aufforderungen wie $\lambda \alpha \beta \dot{\omega} v$ à $\nu a ́ \gamma v \omega \vartheta \iota$ Bd. I S. 105, 15,

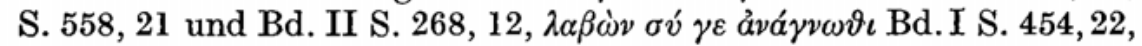

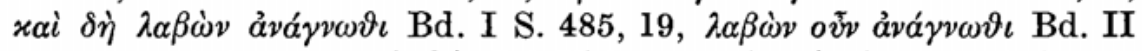

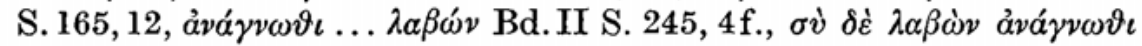

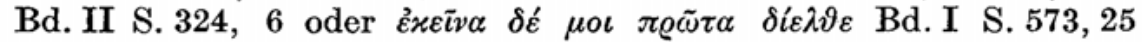
gelten ja natürlich dem Leser, nichts jedoch hindert uns anzunehmen, $\mathrm{da} \beta$ sie auch den um Eusebius herumstehenden Diakonen galten. Das von einem Diakon Vorgelesene wird von anderen nachgeschrieben. Nach Beendigung jeder solchen Vorlesung macht Eusebius seine (manchmal zu Homilien anwachsenden) Bemerkungen dazu, die ebenfalls gleich nachgeschrieben werden. So etwa haben wir uns m. E. das Zustandekommen dieses umfangreichen Werkes vorzustellen.

1) Ob sie Aristoteles' Schriften enthielt, ist fraglich, jedenfalls verwendet Eus. zur Darstellung der Lehre des Aristoteles (Buch XV Kap. 2-13) nicht dessen Originalwerke, sondern Schriften des Aristocles, Atticus, Plotin, Porphyrius. 